\title{
Globalization, free markets and investor protection
}

\author{
Theophilus A. Lartey • Albert Danso* \\ Leicester Castle Business School, De Montfort University, Leicester, UK
}

Received: 1 August 2019

Revised: 27 September 2019

Accepted: 3 October 2019

\begin{abstract}
Using data from 29 countries, this paper is the first attempt to examine whether economic, political and social integration explain how well investors, both shareholders and creditors, are protected from expropriation by firms. We show that: (i) globalization drives both shareholder and creditor protection; (ii) least restrictive markets rather than paternalistic markets matter particularly for shareholders' protection; (iii) the globalization-protection nexus favoured only creditors during the crisis; and (iv) our result significantly holds for OECD-member countries.
\end{abstract}

Keywords: globalization; shareholder protection; creditor protection; free market; financial crisis JEL Classification Codes: E02, F30, G01, K22, O16, P50

\section{Introduction}

While Investor Protection (IP) delineates the extent of legal protection afforded to investors to mitigate expropriation by controlling shareholders or managers (Shleifer and Wolfenzon, 2002), globalization epitomizes global integration driven by the natural evolution of markets, economies, cultures, technologies and governance (Sobol et al., 2018). Globalization of countries and markets represents a core development in the last half century (Steenkamp, 2019), though it is analogous to a double-edged sword (Djelic and Quack, 2018). Indeed, increasing cross-border holdings and external portfolio sizes of countries have ratified the surge of economic-financial integration among countries (Lane and Milesi-Ferretti, 2007).

Extant literature primarily considers economic integration, even though political and social integration are effective towards improving risk-sharing and protection (Flood et al., 2012). Political and social integration facilitate growth (Alesina et al., 2000), the international enforceability of contracts (Balli et al., 2018), and the efficient execution of cross-border mergers and acquisitions (Weber and Camerer, 2003). Economic integration is usually the outcome of political arrangements and social proximity. Indeed, the European Monetary Union's institution of a common currency uniquely motivates an investigation into the impact of economic and socio-political integration. Yet, no evidence exists on whether these forms of global integration drive improvements in investor protection laws. Thus, we empirically assess whether, along with economic integration, political and social globalization play roles in smoothing the legal protection of international investors, both shareholders and creditors.

*Corresponding author. E-mail: albert.danso@dmu.ac.uk.

Citation: Lartey, T. A. and Danso, A. (2020) Globalization, free markets and investor protection, Economics and Business Letters, 9(1), 8-13.

DOI: $10.17811 /$ ebl.9.1.2020.8-13 


\section{Data}

\subsection{Data}

We utilize data on financial and macroeconomic aggregates from the World Development Indicators database for 29 countries, combined with shareholder and creditor protection data from the Centre for Business Research, and the KOF globalization index spanning between 1990 and 2013. The choice of the 29 countries and the period are driven by availability of data for this study. The KOF index provides globalization data along economic, social and political lines. Economic globalization characterizes exposure to global market forces; social globalization captures exposure to global media, the spread of ideas, information and people; and political globalization characterizes diffusion of government policies, alliance with international organizations and bilateral relations with other countries (Gygli et al., 2018; Eppinger and Potrafke, 2016).

\subsection{Methodology}

Our baseline model is estimated via the two-stage General Method of Moment (Arellano and Bover, 1995; Blundell and Bond, 1998) estimator specified as:

Stage 1: We regress investor protection on the instruments and other exogenous variables:

$$
\begin{gathered}
P\left(\text { Globalization }_{i, t}=1 \mid \text { Globalization }_{i, t-1} \text {, Inforflows }_{i, t}, Y_{i, t}\right)=\delta_{1}+ \\
\delta_{2} \text { Globalization }_{i, t-1}+\delta_{3} \text { Inforflows }_{i, t}+\delta_{4} Y_{i, t}+v_{i}+\omega_{t}
\end{gathered}
$$

Stage 2: We replace the fitted value of Globalization $_{i, t}$ derived from the first stage with Globalization $i_{i, t}^{*}$ in the main regression (Eq. 2):

$$
\text { Investor_Protection }_{i, t}=\alpha+\beta_{1} \text { Globalization }_{i, t}^{*}+\beta_{2} X_{i, t}+v_{i}+\omega_{t}+\varepsilon_{i, t}
$$

where Investor_Protection is the measure of shareholder and creditor protection, Globalization $_{i, t}$ is the instrumented globalization measure (economic, social, political and aggregate where appropriate), Globalization ${ }_{i, t}^{*}$ is the predicted value of globalization from the first stage regression, Inforflows $s_{i, t}$ is the instrumented information flows variable, $Y_{i t}$ represents the control variables that include Trade (\%GDP) and FDI flows (\%GDP) capturing trade/economic openness; log of income per capita captures the general level of development; CBOE volatility index captures investors' short-term fears about instability and uncertainty in global financial markets; interpolated GINI captures inequality; level of democracy captures the system of government; sovereign lending captures short-term sovereign lending rate in the global economy; urbanization captures population living in urban centres; log of population density captures the effects of knowledge spillovers; $v_{i}$ (and $w_{t}$ ) are the country (and year) effect and $\varepsilon_{i, t}$ is the error term. The choice of the Arellano-Bover/Blundell-Bond two-stage GMM accounts for endogeneity, time-varying endogenous effects, reverse causality and is more fitting for our large sample (i.e. $\mathrm{N}=29$ countries). As instruments, we use the lagged globalization index (specific for each model) and the level of information flows of each country. The intuition is that current-year integration may be positively related to prior-year integration and the country-specific information flows to (from) other jurisdictions. However, we do not suggest that these instruments have a direct economic effect on investor protection, and thus they may be uncorrelated with the error term in the second stage regression. We regress each globalization measure on the country-specific variables (trade, income per capita, CBOE volatility, FDI, GINI, democracy, sovereign lending, urbanization, population-density), and include the predicted variables in the second stage.

\section{Results}

\subsection{Globalization and IP}

Table 1 reports the results of estimating Eq. (1). The coefficients of our four globalization measures on the IP indicators, Shareholder Protection and Creditor Protection, are positive and statistically significant at one percent, hence suggesting that erosion of boundaries and 
integration of economies/markets, cultures, technologies and governance drive improvements in IP. Economically, a point increase in globalization induces an increase in Shareholder Protection and Creditor Protection by 0.08 (0.08) points, representing a 5.3\% (5.3\%) increase relative to the sample standard deviation for aggregate globalization of 15.1.

Table 1. Globalization and IP (GMM).

\begin{tabular}{|c|c|c|c|c|c|c|c|c|}
\hline & \multicolumn{4}{|c|}{ "Shareholder Protection } & \multicolumn{4}{|c|}{ Creditor Protection } \\
\hline & (1) & (2) & (3) & (4) & (5) & (6) & (7) & (8) \\
\hline Economic & $0.057^{* * *}$ & & & & $0.048^{* * *}$ & & & \\
\hline Globalization & $(0.012)$ & & & & $(0.011)$ & & & \\
\hline Social Globalization & & $\begin{array}{r}0.043^{* * *} \\
(0.012)\end{array}$ & & & & $\begin{array}{r}0.052^{* * *} \\
(0.016)\end{array}$ & & \\
\hline Political Globalization & & & $\begin{array}{c}0.028^{* * * *} \\
(0.007)\end{array}$ & & & & $\begin{array}{r}0.028^{* * *} \\
(0.009)\end{array}$ & \\
\hline Aggregate & & & & $0.080^{* * *}$ & & & & $0.080^{* * *}$ \\
\hline Globalization & & & & $(0.014)$ & & & & $(0.015)$ \\
\hline Trade (\%GDP) & $-0.427^{*}$ & 0.075 & $0.381^{* *}$ & -0.209 & 0.304 & $0.632^{* *}$ & $0.999^{* * *}$ & 0.408 \\
\hline & $(0.253)$ & $(0.199)$ & $(0.169)$ & $(0.211)$ & $(0.306)$ & $(0.300)$ & $(0.254)$ & $(0.292)$ \\
\hline Income per capita & $0.733^{* * *}$ & $0.578^{* * *}$ & $1.032^{* * *}$ & $0.579^{* * *}$ & -0.262 & $-0.556^{* *}$ & -0.008 & $-0.461^{* *}$ \\
\hline & $(0.150)$ & $(0.219)$ & $(0.146)$ & $(0.175)$ & $(0.169)$ & $(0.257)$ & $(0.175)$ & $(0.199)$ \\
\hline CBOE Volatility & 0.009 & 0.004 & 0.008 & 0.003 & 0.007 & 0.001 & 0.006 & 0.002 \\
\hline & $(0.009)$ & $(0.010)$ & $(0.010)$ & $(0.009)$ & $(0.012)$ & $(0.012)$ & $(0.012)$ & $(0.012)$ \\
\hline$F D I(\% G D P)$ & 0.034 & 0.038 & 0.131 & -0.017 & $-0.382^{* * *}$ & $-0.431^{* * *}$ & $-0.308^{* *}$ & $-0.456^{\text {**** }}$ \\
\hline & $(0.095)$ & $(0.099)$ & $(0.094)$ & $(0.094)$ & $(0.134)$ & $(0.120)$ & $(0.128)$ & $(0.125)$ \\
\hline Interpolated GINI & 0.001 & $0.039^{* * *}$ & $0.025^{* * *}$ & $0.034^{* * *}$ & $-0.040^{* * * 1}$ & 0.001 & -0.018 & -0.010 \\
\hline & $(0.007)$ & $(0.009)$ & $(0.008)$ & $(0.007)$ & $(0.012)$ & $(0.015)$ & $(0.012)$ & $(0.012)$ \\
\hline Level of Democracy & -0.026 & -0.001 & -0.039 & -0.170 & 0.255 & 0.234 & 0.216 & 0.091 \\
\hline & $(0.245)$ & $(0.258)$ & $(0.262)$ & $(0.255)$ & $(0.253)$ & $(0.245)$ & $(0.263)$ & $(0.248)$ \\
\hline Sovereign Lending & $-0.156^{* * *}$ & $-0.182^{* * * *}$ & $-0.148^{* * * *}$ & $-0.151^{* * *}$ & -0.010 & -0.033 & 0.001 & -0.000 \\
\hline & $(0.035)$ & $(0.034)$ & $(0.033)$ & $(0.032)$ & $(0.043)$ & $(0.043)$ & $(0.043)$ & $(0.041)$ \\
\hline Urbanization & $-0.039^{* * *}$ & $-0.029^{* * * *}$ & $-0.036^{* * *}$ & $-0.040^{* * *}$ & $0.022^{* *}$ & $0.031^{* * *}$ & $0.024^{* * *}$ & $0.020^{* * *}$ \\
\hline & $(0.008)$ & $(0.007)$ & $(0.007)$ & $(0.007)$ & $(0.008)$ & $(0.007)$ & $(0.008)$ & $(0.008)$ \\
\hline Population-Density & $0.443^{* * *}$ & $0.284^{* * *}$ & $0.223^{* * * *}$ & $0.253^{* * * *}$ & $0.403^{* * *}$ & $0.243^{* * *}$ & $0.198^{* *}$ & $0.227^{* * *}$ \\
\hline & $(0.068)$ & $(0.075)$ & $(0.082)$ & $(0.072)$ & $(0.071)$ & $(0.080)$ & $(0.082)$ & $(0.073)$ \\
\hline _cons & $-8.270^{* * * *}$ & $-7.197^{* * * *}$ & $-10.373^{* * * *}$ & $-7.299^{* * *}$ & -3.268 & -1.268 & $-5.139^{*}$ & -2.011 \\
\hline & $(1.953)$ & $(2.248)$ & $(1.867)$ & $(1.996)$ & $(2.729)$ & $(3.308)$ & $(2.692)$ & $(2.836)$ \\
\hline Country Effects & Yes & Yes & Yes & Yes & Yes & Yes & Yes & Yes \\
\hline Year Effects & Yes & Yes & Yes & Yes & Yes & Yes & Yes & Yes \\
\hline Observations & 696 & 696 & 696 & 696 & 696 & 696 & 696 & 696 \\
\hline$K-P W F$ statistic & 657.030 & 568.467 & 333.124 & 639.980 & 657.030 & 568.467 & 333.124 & 639.980 \\
\hline K-P LM statistic & 77.504 & 97.743 & 57.260 & 75.959 & 77.504 & 97.743 & 57.260 & 75.959 \\
\hline Hansen J statistic & 1.809 & 1.632 & 1.552 & 1.743 & 0.069 & 0.017 & 0.080 & 0.037 \\
\hline Hansen J p-value & 0.179 & 0.201 & 0.213 & 0.187 & 0.793 & 0.897 & 0.778 & 0.847 \\
\hline
\end{tabular}

Notes. Standard error robust to heteroscedasticity in parentheses and clustering at country and year level; *Indicates $10 \%$ significance; ${ }^{* *}$ Indicates $5 \%$ significance; ${ }^{* * *}$ Indicates $1 \%$ significance.

\subsection{Globalization, free markets and IP}

Higher-regulated markets may favour equality over globalization and growth (Dorn et al., 2018). Hence, to segregate the role of legal systems, we examine whether effectively protecting investors necessitates not only increased globalization but also a free-market system. We capture free-market capitalism by the Economic Freedom Index, which measures the extent to which an economy is free of regulation (Gwartney and Lawson, 2003). In Table 2, we observe a significant impact of globalization on Shareholder Protection but an insignificant one on Creditor Protection. Interestingly, the coefficient of globalization is positive only when the interaction variable and the free market variable enter the estimation model simultaneously. The coefficients of globalization and free markets also increase in magnitude if the interaction term is included. Nevertheless, the coefficients on all interaction variables are negative and statistically significant. Overall, our finding indicates that globalization drives investor protection. 
Table 2. Globalization and IP: The Role of Free Markets (GMM).

\begin{tabular}{|c|c|c|c|c|c|c|}
\hline & \multicolumn{3}{|c|}{ Shareholder Protection } & \multicolumn{3}{|c|}{ Creditor Protection } \\
\hline & (1) & $(2)$ & (3) & (4) & (5) & $(6)$ \\
\hline Aggregate Globalization & $\begin{array}{c}-0.014^{* * *} \\
(0.005)\end{array}$ & $\begin{array}{r}-0.045^{* * *} \\
(0.014)\end{array}$ & $\begin{array}{c}0.222^{* * * *} \\
(0.051)\end{array}$ & $\begin{array}{r}-0.010 \\
(0.005)\end{array}$ & $\begin{array}{r}-0.055 \\
(0.013)\end{array}$ & $\begin{array}{r}0.061 \\
(0.039)\end{array}$ \\
\hline Free Markets & $\begin{array}{r}0.632^{* * *} \\
(0.078)\end{array}$ & & $\begin{array}{r}2.931^{* * * *} \\
(0.489)\end{array}$ & $\begin{array}{r}0.677^{* * *} \\
(0.085)\end{array}$ & & $\begin{array}{l}1.276^{* * * *} \\
(0.360)\end{array}$ \\
\hline Agg. Globalization $*$ Free & & $0.006^{* * *}$ & $\begin{array}{r}-0.034^{* * * *} \\
(0007)\end{array}$ & & $0.009^{* * *}$ & -0.009 \\
\hline cons & $\begin{array}{c}1.896^{* * *} \\
(0.463)\end{array}$ & $\begin{array}{c}5.432^{* * *} \\
(0.424)\end{array}$ & $\begin{array}{r}-13.635^{* * *} \\
(3.338)\end{array}$ & $\begin{array}{l}0.954^{* *} \\
(0.458)\end{array}$ & $\begin{array}{c}5.222^{* * * *} \\
(0.339)\end{array}$ & $\begin{array}{r}-3.099 \\
(2.507) \\
\end{array}$ \\
\hline Country Effects & Yes & Yes & Yes & Yes & Yes & Yes \\
\hline Year Effects & Yes & Yes & Yes & Yes & Yes & Yes \\
\hline Observations & 642 & 642 & 642 & 642 & 642 & 642 \\
\hline$K-P W F$ statistic & 910.239 & 147.919 & 147.834 & 910.239 & 147.919 & 147.834 \\
\hline$K-P$ LM statistic & 167.265 & 191.140 & 68.786 & 167.265 & 191.140 & 68.786 \\
\hline Hansen J statistic & 0.159 & 1.228 & 0.409 & 0.001 & 0.076 & 0.020 \\
\hline Hansen J p-value & 0.690 & 0.268 & 0.523 & 0.971 & 0.783 & 0.888 \\
\hline
\end{tabular}

Notes. Standard error robust to heteroscedasticity in parentheses and clustering at country and year level; *Indicates $10 \%$ significance; ${ }^{* * *}$ Indicates $5 \%$ significance; ${ }^{* * *}$ Indicates $1 \%$ significance.

Table 3. Globalization and IP: Does crisis matter? (GMM).

\begin{tabular}{|c|c|c|c|c|c|c|}
\hline & \multicolumn{2}{|c|}{ Pre-Crisis } & \multicolumn{2}{|c|}{ Crisis } & \multicolumn{2}{|c|}{ Post-Crisis } \\
\hline & $\begin{array}{c}\text { Shareholder } \\
\text { Protection }\end{array}$ & $\begin{array}{c}\text { Creditor } \\
\text { Protection }\end{array}$ & $\begin{array}{c}\text { Shareholder } \\
\text { Protection }\end{array}$ & $\begin{array}{c}\text { Creditor } \\
\text { Protection }\end{array}$ & $\begin{array}{c}\text { Shareholder } \\
\text { Protection }\end{array}$ & $\begin{array}{c}\text { Creditor } \\
\text { Protection }\end{array}$ \\
\hline & (1) & (2) & (3) & (4) & $(5)$ & (6) \\
\hline Aggregate & $0.084^{* * * *}$ & $0.081^{* * *}$ & 0.006 & $0.055^{* * *}$ & $0.041^{* * *}$ & $0.069^{* * * *}$ \\
\hline Globalization & $(0.014)$ & $(0.022)$ & $(0.020)$ & $(0.018)$ & $(0.017)$ & $(0.012)$ \\
\hline Trade (\%GDP) & $-0.513^{*}$ & 0.248 & $-0.474^{*}$ & $0.559^{* * *}$ & -0.363 & $0.766^{\text {**** }}$ \\
\hline & $(0.272)$ & $(0.424)$ & $(0.265)$ & $(0.282)$ & $(0.254)$ & $(0.274)$ \\
\hline Income per & $0.445^{* *}$ & -0.332 & $0.825^{* * *}$ & $-0.480^{* * *}$ & $0.358^{*}$ & $-0.524^{* * * *}$ \\
\hline capita & $(0.200)$ & $(0.293)$ & $(0.263)$ & $(0.232)$ & $(0.188)$ & (0.183) \\
\hline CBOE & 0.006 & 0.013 & 0.033 & 0.010 & -0.018 & -0.000 \\
\hline Volatility & $(0.012)$ & $(0.018)$ & $(0.067)$ & $(0.088)$ & $(0.020)$ & $(0.022)$ \\
\hline$F D I(\% G D P)$ & $-0.351^{* *}$ & $-0.758^{* * * *}$ & 0.187 & -0.151 & -0.077 & $-0.405^{* *}$ \\
\hline & $(0.137)$ & $(0.200)$ & $(0.172)$ & $(0.198)$ & $(0.120)$ & $(0.169)$ \\
\hline GINI & $0.067^{* * * *}$ & 0.002 & $-0.032^{* *}$ & $-0.024^{* *}$ & $-0.023^{\text {** }}$ & $-0.014^{* *}$ \\
\hline & (0.009) & $(0.018)$ & $(0.013)$ & $(0.010)$ & $(0.011)$ & $(0.006)$ \\
\hline Level of & $0.695^{* *}$ & 0.157 & $-0.699^{* *}$ & $0.453^{*}$ & $-0.731^{\text {**** }}$ & 0.206 \\
\hline Democracy & $(0.342)$ & $(0.445)$ & $(0.301)$ & $(0.240)$ & $(0.191)$ & $(0.219)$ \\
\hline Sovereign & $-0.105^{* * *}$ & -0.001 & 0.101 & 0.056 & 0.024 & 0.035 \\
\hline Lending & (0.039) & $(0.055)$ & (0.373) & $(0.490)$ & $(0.145)$ & $(0.155)$ \\
\hline Urbanization & $-0.036^{* * *}$ & $0.035^{* * *}$ & $-0.029^{* * *}$ & 0.006 & $-0.020^{* *}$ & 0.012 \\
\hline & $(0.008)$ & $(0.012)$ & $(0.010)$ & $(0.011)$ & $(0.008)$ & $(0.009)$ \\
\hline Population- & -0.025 & -0.014 & $0.151^{*}$ & $0.427^{* * *}$ & $0.114^{*}$ & $0.387^{* * *}$ \\
\hline Density & $(0.074)$ & $(0.126)$ & $(0.087)$ & $(0.047)$ & $(0.068)$ & $(0.062)$ \\
\hline _cons & -2.843 & 0.013 & -0.077 & -3.560 & 3.121 & $-4.222^{*}$ \\
\hline & (2.201) & $(3.844)$ & (3.802) & $(3.929)$ & $(2.559)$ & $(2.177)$ \\
\hline Country Effects & Yes & Yes & Yes & Yes & Yes & Yes \\
\hline Year Effects & Yes & Yes & Yes & Yes & Yes & Yes \\
\hline Observations & 493 & 493 & 87 & 87 & 145 & 145 \\
\hline$K-P W F$ & 331.485 & 331.485 & 168.007 & 168.007 & 152.767 & 152.767 \\
\hline statistic & & & & & & \\
\hline $\begin{array}{l}\text { K-P LM } \\
\text { statistic }\end{array}$ & 48.378 & 48.378 & 17.228 & 17.228 & 18.334 & 18.334 \\
\hline $\begin{array}{l}\text { Hansen J } \\
\text { statistic }\end{array}$ & 1.314 & 0.122 & 1.608 & 0.447 & 0.343 & 0.126 \\
\hline $\begin{array}{l}\text { Hansen Jp- } \\
\text { value }\end{array}$ & 0.252 & 0.727 & 0.205 & 0.504 & 0.558 & 0.723 \\
\hline
\end{tabular}

Notes. Standard error robust to heteroscedasticity in parentheses and clustering at country and year level; ${ }^{*}$ Indicates $10 \%$ significance; ${ }^{* *}$ Indicates $5 \%$ significance; ${ }^{* * *}$ Indicates $1 \%$ significance. 


\subsection{Globalization and IP: Does crisis matter?}

Evidence contends that earnings quality declined during the financial crisis particularly for countries with poor investor protection (Persakis and Iatridis, 2015). In Table 3, we examine the effects of the global downturn on the globalization-protection nexus. Our results indicate that globalization had a positive but immaterial effect on shareholder protection but not on creditor protection during the crisis. This, however, reversed post crisis. This finding indicates that legal protection for creditors is more closely related to underlying economic activity and reaffirms the necessity of government intervention and regulation to protect creditors during the financial crisis (Liu et al., 2016).

\subsection{Globalization and IP: Does OECD membership matter?}

Table 4 examines whether being a member of the Organization for Economic Co-operation and Development (OECD) matters for our globalization-protection nexus. The OECD aims to promote policies that enhance economic and social well-being globally (Allin and Hand, 2017). Our results show that, for OECD-member countries, characterized by a common goal towards driving economic, commercial/financial, social and environmental change, globalization significantly promotes protection of both shareholders and creditors (at $1 \%$ level). For nonOECD-member countries, we observe an insignificant impact of globalization on investor protection, hence suggesting that, when countries follow a common goal, they are more likely to provide a law enforcement system and legal structures that effectively protect property and enforcement rights of owners and creditors.

Table 4. Globalization and IP: Does OECD membership matter? (GMM).

\begin{tabular}{|c|c|c|c|c|}
\hline & \multicolumn{2}{|c|}{ OECD Member } & \multicolumn{2}{|c|}{ "Non-OECD Member } \\
\hline & $\begin{array}{l}\text { Shareholder } \\
\text { Protection }\end{array}$ & $\begin{array}{c}\text { Creditor } \\
\text { Protection }\end{array}$ & $\begin{array}{l}\text { Shareholder } \\
\text { Protection }\end{array}$ & $\begin{array}{c}\text { Creditor } \\
\text { Protection }\end{array}$ \\
\hline Aggregate Globalization & $\begin{array}{r}0.083^{* * *} \\
(0.022)\end{array}$ & $\begin{array}{c}0.053^{* * *} \\
(0.014)\end{array}$ & $\begin{array}{r}0.024 \\
(0.027)\end{array}$ & $\begin{array}{r}0.037 \\
(0.035)\end{array}$ \\
\hline Trade (\%GDP) & $\begin{array}{r}-0.301 \\
(0.309)\end{array}$ & $\begin{array}{r}0.135 \\
(0.286)\end{array}$ & $\begin{array}{c}1.681^{* * * *} \\
(0.495)\end{array}$ & $\begin{array}{r}0.087 \\
(0.895)\end{array}$ \\
\hline Income per capita & $\begin{array}{r}0.960^{* * *} \\
(0.266)\end{array}$ & $\begin{array}{r}-0.553^{* *} \\
(0.239)\end{array}$ & $\begin{array}{l}1.035^{\text {** }} \\
(0.509)\end{array}$ & $\begin{array}{l}-0.286 \\
(0.596)\end{array}$ \\
\hline CBOE Volatility & $\begin{array}{r}0.005 \\
(0.009)\end{array}$ & $\begin{array}{r}0.009 \\
(0.011)\end{array}$ & $\begin{array}{r}0.007 \\
(0.014)\end{array}$ & $\begin{array}{r}-0.001 \\
(0.022)\end{array}$ \\
\hline$F D I(\% G D P)$ & $\begin{array}{l}-0.026 \\
(0.105)\end{array}$ & $\begin{array}{r}-0.390^{* * *} \\
(0.138)\end{array}$ & $\begin{array}{r}-0.129 \\
(0.153)\end{array}$ & $\begin{array}{r}-0.249 \\
(0.285)\end{array}$ \\
\hline GINI & $\begin{array}{r}-0.044 \\
(0.028)\end{array}$ & $\begin{array}{r}-0.057^{* *} \\
(0.023)\end{array}$ & $\begin{array}{c}0.014^{*} \\
(0.008)\end{array}$ & $\begin{array}{r}0.013 \\
(0.013)\end{array}$ \\
\hline Level of Democracy & $\begin{array}{c}-0.888^{* * * *} \\
(0.200)\end{array}$ & $\begin{array}{r}0.305 \\
(0.263)\end{array}$ & $\begin{array}{r}0.625 \\
(0.445)\end{array}$ & $\begin{array}{r}-0.768 \\
(0.556)\end{array}$ \\
\hline Sovereign Lending & $\begin{array}{r}-0.127^{* * * *} \\
(0.031)\end{array}$ & $\begin{array}{r}0.053 \\
(0.039)\end{array}$ & $\begin{array}{r}-0.094 \\
(0.063)\end{array}$ & $\begin{array}{r}-0.230^{* * * *} \\
(0.078)\end{array}$ \\
\hline Urbanization & $\begin{array}{l}-0.024^{*} \\
(0.013)\end{array}$ & $\begin{array}{r}0.019 \\
(0.012)\end{array}$ & $\begin{array}{r}-0.005 \\
(0.015)\end{array}$ & $\begin{array}{r}0.021 \\
(0.022)\end{array}$ \\
\hline Population-Density & $\begin{array}{l}0.424^{* *} \\
(0.169)\end{array}$ & $\begin{array}{r}0.186 \\
(0.114)\end{array}$ & $\begin{array}{c}0.904^{* * * *} \\
(0.152)\end{array}$ & $\begin{array}{r}-0.183 \\
(0.221)\end{array}$ \\
\hline _cons & $\begin{array}{r}-11.694^{* * *} \\
(3.664)\end{array}$ & $\begin{array}{r}4.015 \\
(3.341) \\
\end{array}$ & $\begin{array}{r}-27.983^{* * *} \\
(7.514) \\
\end{array}$ & $\begin{array}{r}7.329 \\
(10.078) \\
\end{array}$ \\
\hline Country Effects & Yes & Yes & Yes & Yes \\
\hline Year Effects & Yes & Yes & Yes & Yes \\
\hline Observations & 175 & 175 & 521 & 521 \\
\hline$K-P W F$ statistic & 726.749 & 726.749 & 115.337 & 115.337 \\
\hline$K-P$ LM statistic & 38.123 & 38.123 & 32.047 & 32.047 \\
\hline Hansen J statistic & 0.091 & 0.070 & 2.855 & 0.060 \\
\hline Hansen J p-value & 0.763 & 0.791 & 0.091 & 0.807 \\
\hline
\end{tabular}

Notes. Standard error robust to heteroscedasticity in parentheses and clustering at country and year level; "Indicates $10 \%$ significance; ${ }^{* *}$ Indicates $5 \%$ significance; ${ }^{* * *}$ Indicates $1 \%$ significance. 


\section{Conclusion}

Using panel data for 29 countries, we shed new light on the key role of globalization on investor protection. We observe that globalization explains how well investors, both shareholders and creditors, are protected particularly for least restrictive markets and OECD-member countries. Also, the globalization-protection nexus favoured only creditors during the crisis. Our findings have key policy implications. The work also complements evidence on the relation between globalization and economic growth, international enforceability of contracts, and cross-border mergers and acquisitions (Balli et al., 2018).

\section{References}

Alesina, A., Spolaore, E., and Wacziarg, R. (2000) Economic integration and political disintegration, American Economic Review, 90, 1276-1296.

Allin, P., and Hand, D. J. (2017) New statistics for old? - measuring the wellbeing of the UK, Journal of the Royal Statistical Society: Series A (Statistics in Society), 180, 3-43.

Arellano, M., and Bover, O. (1995). Another look at the instrumental variable estimation of error-components models, Journal of Econometrics, 68(1), 29-51.

Balli, F., Pericoli, F. M., and Pierucci, E. (2018) Globalization and international risk-sharing: The role of social and political integration, European Journal of Political Economy, 55, 324345.

Blundell, R., and Bond, S. (1998) Initial conditions and moment restrictions in dynamic panel data models, Journal of Econometrics, 87(1), 115-143.

Djelic, M., and Quack, S. (2018) Globalization and business regulation, Annual Review of Sociology, 44, 123-143.

Dorn, F., Fuest, C., and Potrafke, N. (2018) Globalization and income inequality revisited, CESifo Working Paper Series, No. 6859.

Eppinger, P. and Potrafke, N. (2016) Did globalisation influence credit market deregulation?, The World Economy, 39, 426-443.

Flood, R. P., Marion, N. P., and Matsumoto, A. (2012) International risk sharing during the globalization era, Canadian Journal of Economics, 45, 394-416.

Gwartney, J., and Lawson, R. (2003) The concept and measurement of economic freedom, European Journal of Political Economy, 19, 405-430.

Gygli, S., Haelg, F., Potrafke, N., and Sturm, J. (2018) The KOF globalisation index-revisited, The Review of International Organizations, 1-32.

Lane, P. R., and Milesi-Ferretti, G. M. (2007) The external wealth of nations mark II: Revised and extended estimates of foreign assets and liabilities, 1970-2004, Journal of International Economics, 73, 223-250.

Liu, L., Zhang, G., and Fang, Y. (2016) Bank credit default swaps and deposit insurance around the world, Journal of International Money and Finance, 69, 339-363.

Persakis, A., and Iatridis, G. E. (2015) Cost of capital, audit and earnings quality under financial crisis: A global empirical investigation, Journal of International Financial Markets, Institutions and Money, 38, 3-24.

Shleifer, A., and Wolfenzon, D. (2002) Investor protection and equity markets, Journal of Financial Economics, 66, 3-27.

Sobol, K., Cleveland, M., and Laroche, M. (2018) Globalization, national identity, biculturalism and consumer behavior: A longitudinal study of Dutch consumers, Journal of Business Research, 82, 340-353.

Steenkamp, J. (2019) The uncertain future of globalization: Implications for global consumer culture and global brands, International Marketing Review, in press.

Weber, R. A., and Camerer, C. F. (2003) Cultural conflict and merger failure: An experimental approach, Management Science, 49, 400-415. 